\title{
Mycoplasma fastidiosum: a New Species from Horses
}

\author{
R. M. LEMCKE AND J. POLAND \\ Agricultural Research Council, Institute for Research on Animal Diseases, Compton, Newbury, Berkshire, ${ }^{1}$ \\ and Royal Veterinary College, London, NW1 OTU ${ }^{2}$ England
}

\begin{abstract}
The morphology, growth characteristics, biochemical activities, and serological relationships of a group of eight slow glucose-metabolizing mycoplasmas isolated from nasopharyngeal swabs from horses are described. Representatives of the group possessed a triple-layered limiting membrane and had the essential cultural and biological properties of mycoplasmas. They were highly filamentous, but unusual morphological features were the twisting of the filaments and the regular diagonal striations revealed by electron microscopy. Also notable were the somewhat fastidious nutritional requirements of the slow glucose-metabolizing mycoplasmas and their susceptibility to erythromycin $(0.1 \mu \mathrm{g} / \mathrm{ml})$. Serologically, a representative strain, 4822, was distinct from strains of 58 species of Mycoplasma and of Spiroplasma citri when tested by the disk growth inhibition test or the indirect fluorescent-antibody test. A cross-reaction with certain strains of $M$. pulmonis was detected in some growth inhibition tests on a medium (LH agar) subsequently shown to be suboptimal for growth. Comparison of 4822 with several strains of $M$. pulmonis in growth inhibition, metabolism inhibition, and indirect fluorescent-antibody tests with HuS medium failed to show any cross-reactions. Thus, serological methods based on inhibition of growth or metabolism must be used with caution when identifying nutritionally fastidious mycoplasmas that are likely to be inhibited by nonspecific factors. The evidence presented here suggests that the slow glucose-metabolizing strains constitute a new species, for which the name Mycoplasma fastidiosum is proposed. Strain $4822(=$ NCTC 10180) is the type strain.
\end{abstract}

During a survey of 51 young thoroughbreds in training that were sampled at regular intervals by means of nasopharyngeal swabs, a group of mycoplasmas that slowly metabolized glucose was isolated (15). These isolates were referred to as SGM (slow glucose-metabolizing) strains. They were obtained from $7(14 \%)$ of the 51 horses and were found in only three of the seven stables involved in the survey. On primary isolation, colonies developed slowly and were never isolated when other more rapidly growing mycoplasmas such as Acholeplasma laidlawii and Mycoplasma equirhinis were cultivated from a swab. SGM strains had not been isolated from horses, young or old, in any earlier surveys $(1,9$, 20 ). Their isolation was thought to be due to the use of a medium that particularly favored the growth of these strains and also to preincubation of the swabs before subculture (15).

In the course of serological tests to identify the isolates, a cross-reaction with strain N14, an equine isolate of Mycoplasma pulmonis, was observed in some growth inhibition (GI) and metabolism inhibition (MI) tests on one of the two media used, and the taxonomic status of the SGM strains was therefore reported as uncertain (15). More detailed morphological, biochemical, and serological tests on the SGM strains suggest that they comprise a new species of Mycoplasma.

\section{MATERIALS AND METHODS}

Mycoplasma strains. Eight SGM strains were obtained from nasopharyngeal swabs from seven different horses (15) and given the number 4788, 4791, $4822,4927,4929,4930,5173$, and 5889 , according to the case numbers of the horses. Strain 4822 was taken as the strain representative of the group. The equine mycoplasmas $M$. pulmonis strain N14, $M$. felis strain N29B, Mycoplasma sp. strain equine N3, and $M$. equirhinis strain M432/72 have been described elsewhere $(1,11)$. $M$. equigenitalium strain T37 and $\boldsymbol{M}$. subdolum strains TB and H8360 were obtained from the genital tract of mares $(10,12)$. The strain designations of all the Mycoplasma species used in the serological tests are shown in Table 1.

Media. Two types of media were used. One (HuS) contained Difco PPLO broth $(70 \%$, vol $/ \mathrm{vol}), 25 \%$ aqueous extract of dried yeast $(10 \%$, vol/ vol $)$, heatinactivated human serum $(20 \%$, vol/vol), sodium deoxyribonucleate $(0.002 \%, \mathrm{wt} / \mathrm{vol})$, ampicillin $(300 \mu \mathrm{g} / \mathrm{ml})$, thallium acetate $(0.0005 \%$, wt $/ \mathrm{vol})$, and phenol red $(0.002 \%$, wt/vol). The other $(\mathrm{LH})$ consisted of Hanks balanced salt solution (35\%, vol/vol), Oxoid Hartley digest broth $(30 \%$, vol/vol), $5 \%$ aqueous lactalbumin hydrolysate $(10 \%, \mathrm{vol} / \mathrm{vol}), 25 \%$ dried yeast extract 
( $10 \%, \mathrm{vol} / \mathrm{vol})$, heat-inactivated pig serum $(20 \%$, vol/ $\mathrm{vol})$, ampicillin $(300 \mu \mathrm{g} / \mathrm{ml})$, thallium acetate $(0.0005 \%$, $\mathrm{wt} / \mathrm{vol})$, and phenol red $(0.002 \%$, wt/vol $)$. When these broths were supplemented with either $0.5 \%$ glucose ( $\mathrm{HuSg}$ and $\mathrm{LHg}$ broths) or $0.25 \%$ arginine (HuSa and LHa broths), the $\mathrm{pH}$ was adjusted to 7.8 and 7.0, respectively. HuS and $\mathrm{LH}$ agar were made by adding $1 \%$ Difco Noble agar and adjusting the $\mathrm{pH}$ to about 7.4. For some tests, Oxoid mycoplasma broth base was substituted for Difco PPLO broth in the formulas for HuS media.

Cultivation and purification. Broth was usually dispensed in 2-ml amounts in bijou bottles or in screwcapped vials with a capacity of $3 \mathrm{ml}$. For aerated broth cultures, 2-ml amounts were dispensed in $15-\mathrm{ml}$ screwcapped tubes and rotated at $20 \mathrm{rph}$ in an almost horizontal position. Except for certain biochemical tests, plates wre incubated in $5 \% \mathrm{CO}_{2}$ in $\mathrm{N}_{2}$. All cultures were incubated at $37^{\circ} \mathrm{C}$. Isolates were purified by picking single, isolated colonies into broth. After incubation, dilutions of the broths were replated on HuS agar to obtain isolated colonies. The whole procedure was then repeated at least twice more. The representative strain, 4822 , which was examined in must detail, was cloned five times in this manner. Although cultures were not filtered during cloning, isolation from single colonies was repeated twice more than is recommended (18). Moreover, with highly filamentous cultures like those of the SGM strains, it is doubtful whether filtrates would consist of single cells containing one genome. Stock cultures of each $\mathrm{SGM}$ strain in HuS broth were stored at $-70^{\circ} \mathrm{C}$ after titration in $\mathrm{HuSg}$ broth to determine the number of color-changing units (CCU) per milliliter. To test the possibility of reversion to a bacterial form, strain 4822 was cultured on HuS agar free from penicillin and thallium acetate. Five passages were made at 4-day intervals.

Morphology. Broth cultures in the logarithmic phase were centrifuged at $3,500 \times g$ for $30 \mathrm{~min}$, and the deposits were examined by phase-contrast or dark-field microscopy. Films of the deposits were also fixed in methanol, stained with Giemsa stain, and examined by light microscopy. For examination with the electron microscope (Philips EM 300: accelerating voltage of $80 \mathrm{kV}$ ), centrifuged deposits were fixed, sectioned, and stained with ruthenium red as described by Howard and Gourlay (7) or were fixed with 3\% glutaraldehyde in $0.1 \mathrm{M}$ phosphate buffer and stained with 2 or $6 \%$ ammonium molybdate. Negatively stained preparations were also made by touching carbon-collodion copper grids onto 4-day-old colonies of strain 4822 on HuS agar, fixing, and staining with $2 \%$ sodium phosphotungstate ( $\mathrm{pH} \mathrm{7.2)} \mathrm{for} \mathrm{about} 30 \mathrm{~s}$.

Filtration studies. Four-day-old cultures in $\mathrm{HuSg}$ broth were passaged in sequence through membrane filters with average pore diameters of $650,450,220$, 300 , and $100 \mathrm{~nm}$ (Millipore Corp.) at positive pressure of 50 to $10 \mathrm{lb} / \mathrm{in}^{2}\left(34.45\right.$ to $\left.68.9 \mathrm{kN} / \mathrm{m}^{2}\right)$. Colony counts, with $100.02-\mathrm{ml}$ drops per dilution, were carried out before and after filtration.

Biochemical tests. The ability of SGM strains 4822 and 4788 to utilize glucose, arginine, and urea was tested in HuS broth supplemented with the appropriate substrate: $1 \%$ glucose $(\mathrm{pH}$ of medium adjusted to 7.8 ), $0.5 \%$ arginine ( $\mathrm{pH}$ adjusted to 7.0 ), or $1 \%$ urea
(pH adjusted to 6.2). Volumes $(2 \mathrm{ml})$ of these three media in bijou bottles were inoculated with $0.1 \mathrm{ml}$ of a $10^{-2}$ dilution of a test culture grown in HuS broth without substrate. Tests were performed in triplicate. As a control for each type of medium, substrate-free $\mathrm{HuS}$ broth was adjusted to $\mathrm{pH} 7.8,7.0$, or 6.3. Uninoculated media with and without substrate were included in each test. Besides the SGM strains under test, mycoplasmas with known biochemical activities were included as controls: $M$. pulmonis strains Kon and N14 for glucose utilization, $M$. subdolum strain TB for arginine degradation, and the bovine Ureaplasma strains T44 and T74 (8) for hydrolysis of urea. The ability to hydrolyze urea was also tested in a medium specifically designed for Ureaplasma species: U3 medium at pH 6.2 (6). For the test, the concentration of urea was raised to $1 \%$, and medium without the substrate was included as a control.

Catalase and oxidase productions were tested on 5day-old colonies, and phosphatase production was tested on 3-, 7- and 14-day-old colonies, on HuS agar (2). Tellurite reduction was determined on $\mathrm{HuS}$ agar without phenol red but containing potassium tellurite. at 0.005 and $0.002 \%$ (wt/vol) and in HuS broth con-. taining $0.002 \%$ potassium tellurite. Plates were incubated aerobically and anaerobically. For testing reduction of 2,3,5-triphenyl tetrazolium chloride, the substrate was added to HuS broth or agar at $0.005 \%$ (wt) vol). Agar cultures were incubated aerobically and anaerobically; all cultures were protected from light. Hemadsorption of sheep and guinea pig erythrocytes (R. A. Del Giudice and R. Pavia, Bacteriol. Proc., p. $71,1964)$ was tested on 5-day-old colonies on HuS agar. Hemolysis was tested by the method of Aluotto et al. (2). Known positive cultures were included in all of the tests cited.

strains 4822 and 4788 were determined by the method of Edward (4), except that the basal medium comprised HuS agar without serum. Inhibition of growth by sodium polyanethol sulfonate and by digitonin was tested on HuS agar by the disk method (5).

Susceptibility to antimicrobial drugs. The susceptibilities of strain 4822 to thallous acetate, amphotericin (Squibb Ltd., Twickenham, England), and erythromycin were determined in HuS broth by the method of Lewis and Poland (13). Strain 4822 was also tested on HuS agar against a range of antimicrobial agents incorporated in dried filter paper disks (Oxoid).

Serological methods. Disk GI tests on agar were performed by the method of Clyde (3), but plates were kept overnight at room temperature before incubation at $37^{\circ} \mathrm{C}$ to assist diffusion of antiserum from the disks. MI was carried out by a modification of the method of Taylor-Robinson et al. (19), in which a 4-drop system was used with a unit volume of $0.05 \mathrm{ml}$ and a final concentration of $1 \%$ guinea pig serum in each well. Wells were covered with sterile liquid paraffin, and the plates were sealed with adhesive tape. GI and MI tests were carried out both in HuS and LH media. The indirect fluorescent-antibody (IFA) technique (16) was carried out on 3-day-old colonies on HuS agar and with fluorescent-labeled anti-rabbit or anti-goat serum (Nordic Diagnostic, Fraberg Ltd., England).

Antiserum production. Antiserum to strain 4822 was prepared in two rabbits, $A$ and $B$, after the strain had been cloned. Antigen for immunization was grown 
in aerated LHg broth because strain 4822 failed to grow in a rabbit infusion broth supplemented with rabbit serum. Washed organisms from $50 \mathrm{ml}$ of culture that contained at least $10^{8} \mathrm{CCU} / \mathrm{ml}$ before harvesting were inoculated intramuscularly in an equal volume of Freund incomplete adjuvant. One month later, the same amount of antigen was administered intravenously. A similar intravenous inoculation was repeated at weekly intervals until an antiserum of high titer was produced. The sources of antisera against recognized species of Mycoplasma are given in Table 1. All sera used in GI tests were tested against their homologous species to ensure that satisfactory inhibition zones $(>2 \mathrm{~mm})$ were produced. Zones of partial inhibition less than $2 \mathrm{~mm}$ in diameter were not considered significant. The homologous reactions of all antisera used in the IFA test were also checked.

\section{RESULTS}

Growth characteristics. Isolated colonies of the eight SGM strains were of the typical "fried egg" morphology on both HuS and LH agars and produced a waxy film after incubation for several days.

On primary isolation, colonies developed more quickly and were slightly larger when incubated in $5 \% \mathrm{CO}_{2}$ in $\mathrm{N}_{2}$ rather than in air. Colonies failed to develop in a strictly anaerobic atmosphere of $5 \% \mathrm{CO}_{2}$ in $\mathrm{H}_{2}$. When standard amounts from the fluid medium in which swabs were incubated were transferred to solid media (15), colonies of SGM mycoplasmas were found to be

TABLE 1. Mycoplasma strains and antisera used in serological tests

\begin{tabular}{|c|c|c|}
\hline Species & Strain(s) & Antiserum source(s) ${ }^{a}$ \\
\hline \multicolumn{3}{|l|}{ Glycolytic } \\
\hline M. pneumoniae & FH & $\mathrm{LI} ; \mathrm{NIH}^{b}$ \\
\hline M. fermentans & PG18; G2 & RVC; LI; NIH ${ }^{b}$ \\
\hline M. moatsii & MK405 & MRL \\
\hline M. mycoides subsp. mycoides & PG1; Gladysdale; G1/61 & MRL; LI \\
\hline$M$. bovirhinis & PG43 & $\mathrm{NIH}^{b} ;$ IRAD \\
\hline M. dispar & $462 / 2 ;$ Gois $^{c}$ & CCAM \\
\hline Mycoplasma sp. group 7 & N29 (PG50) & MRL; CCAM \\
\hline M. bovoculi & M165/69 (NCTC 10141) & MRL \\
\hline M. mycoides subsp. capri & PG3 & MRL; LI \\
\hline M. capricolum & pp. goat & J. Tully \\
\hline M. putrefaciens & KS1 (NCTC 10155) & MRL \\
\hline M. ovipneumoniae & Y98 & G. Jones \\
\hline$M$. conjunctivae & HRC581 & M. F. Barile ${ }^{b}$; MRL \\
\hline M. suipneumoniae & $\mathrm{J}(\mathrm{NCTC} 10110)$ & P. Whittlestone \\
\hline M. hyorhinis & BTS7; GDL; TR32; S & $\mathrm{RVC} ; \mathrm{NIH}^{b}$ \\
\hline M. flocculare & MS42 & $\begin{array}{l}\text { P. Whittlestone } \\
\text { N. Friis }\end{array}$ \\
\hline M. pulmonis & Ash (PG34); M1; Kon; MB; N14 & $\mathrm{NIH}^{b} ; \mathrm{LI}$ \\
\hline M. neurolyticum & KSA, Type A & LI; NIH \\
\hline M. caviae & G122 (NCTC 10126) & A. Hill \\
\hline$M$. canis & PG14; Gois ${ }^{c}$ & $\mathrm{NIH}^{b} ; \mathrm{MRL}$ \\
\hline M. edwardii & PG24 & CCAM \\
\hline M. cynos & H831 (NCTC 10142) & A. Hill \\
\hline M. molare & H542 (NCTC 10144) & CCAM \\
\hline$M$. felis & $\mathrm{CO} ; \mathrm{Gois}^{c} ; \mathrm{N}^{29 \mathrm{~B}^{d}} ; \mathrm{B}^{2} 0^{d}$ & LI \\
\hline M. feliminutum & Ben (NCTC 10159) & CCAM \\
\hline$M$. anatis & 1340 & $\mathrm{NIH}^{b} ; \mathrm{MRL}$ \\
\hline$M$. gallisepticum & S6; X95 (PG31); T & $\mathrm{LI} ; \mathrm{NIH}^{b}$ \\
\hline$M$. synoviae & WVU1853; Lasswade & G. D. Windsor; MRL \\
\hline $\begin{array}{l}\text { Mycoplasma sp. Kleckner's } \\
\text { group D }\end{array}$ & A36; AM & LI \\
\hline M. columborale & MMP4 & MRL \\
\hline M. equigenitalium ${ }^{d}$ & T37 & H. Kirchhoff \\
\hline Mycoplasma sp. equine $\mathrm{N}^{d}$ & $\mathrm{~N} 3 ; \mathrm{N} 11$ & LI \\
\hline M. alvi & Ilsley & IRAD \\
\hline
\end{tabular}

${ }^{a}$ LI, Lister Institute, London (R. Lemcke); NIH, National Institutes of Health, Bethesda, Md. (NIH Research Reference Reagent); RVC, Royal Veterinary College, London (M. Gois or J. Poland); MRL, Mycoplasma Reference Laboratory (R. Leach); IRAD, Institute for Research on Animal Diseases, Compton, Berks (C. Howard or R. N. Gourlay); CCAM, FAO/WHO Collaborating Center for Animal Mycoplasmas, Aarhus, Denmark (E. A. Freundt).

${ }^{b}$ Antisera prepared in donkeys, mules, or burros.

${ }^{c}$ From M. Gois, strain designation unknown.

${ }^{d}$ Species or strains isolated from horses. 
TABLE 1-continued

\begin{tabular}{|c|c|c|}
\hline Species & Strain(s) & Antiserum source(s) \\
\hline \multicolumn{3}{|c|}{$\begin{array}{l}\text { Nonglycolytic, non-arginine-hydro- } \\
\text { lyzing }\end{array}$} \\
\hline M. bovis & PG45 (NCTC 10131); Ab/1 & MRL; IRAD \\
\hline$M$. verecundum & 107 & IRAD \\
\hline M. bovigenitalium & PG11 & $\mathrm{LI} ; \mathrm{NIH}^{b}$ \\
\hline M. agalactiae & PG2; agalactia & $\mathrm{LI} ; \mathrm{NIH}^{b}$ \\
\hline \multicolumn{3}{|l|}{ Arginine-hydrolyzing } \\
\hline M. hominis & Gois ${ }^{c}$; SC4 & LI \\
\hline M. orale & Gois ${ }^{c} ; 837$ & LI \\
\hline M. buccale & CH20247 (NCTC 10136) & $\mathrm{NIH}^{b}$ \\
\hline M. faucium & DC333 & $\mathbf{N I H}^{b}$ \\
\hline M. primatum & Navel; HRC292(NCTC 10163) & LI \\
\hline M. salivarium & Gois $^{c} ;$ B3 & LI \\
\hline M. lipophilum & MaBy & M. F. Barile ${ }^{b}$ \\
\hline M. alkalescens & PG51 (NCTC 10135) & MRL \\
\hline M. arginini & G230; Goisc & $\mathrm{NIH}^{b}$ \\
\hline M. hyosynoviae & $\mathrm{S} 16 ; \mathrm{P} 45$ & CCAM \\
\hline$M$, arthritidis & PG6; PG27; Gois ${ }^{c}$ & $\mathrm{LI} ; \mathrm{NIH}^{b}$ \\
\hline M. maculosum & PG15 & $\mathrm{NIH}^{b}$ \\
\hline M. spumans & PG13 & $\mathrm{NIH}^{b}$ \\
\hline M. gateae & CS & RVC \\
\hline M. gallinarum & Fowl; Gois & LI \\
\hline$M$. iners & M; Gois & LI \\
\hline M. meleagridis & 17529; Gois ${ }^{c}$ & $\mathrm{NIH}^{b}$ \\
\hline M. columbinum & MMP1 & MRL \\
\hline M. equirhinis ${ }^{d}$ & M432/72; H21 & LI \\
\hline M. subdolum ${ }^{d}$ & TB; H8360 & LI \\
\hline M. canadense & 275C (NCTC 10152) & MRL \\
\hline S. citri & Maroc & MRL \\
\hline
\end{tabular}

larger in size and at least 10 to 100 times more numerous on HuS agar than on LH agar. Moreover, colonies were visible after 2 to 4 days of incubation on HuS agar but were not usually detectable until after day 5 on LH agar. Certain strains were more inhibited by $\mathrm{LH}$ agar than others. For example, strain 5889 at an early passage after isolation produced approximately $10^{5}$ fewer colonies on LH than on HuS agar, whereas two other strains, 4930 and 5173, produced $10^{2}$ fewer colonies on LH agar. Some batches of LH medium were more strongly inhibitory than others. No reversion to a bacterial form occurred after five passages on solid medium without inhibitors.

Comparison of the growth of strain 4822 at a high passage level ( $>40$ in vitro passages) and other strains at low passage levels in nonaerated $\mathrm{HuSg}$ and $\mathrm{LHg}$ broths showed that the final number of CCU per milliliter was $10^{1}$ to $10^{4.5}$ times greater, and the decrease in $\mathrm{pH}$ was faster, in HuSg broth. Growth, in terms of number of CCU per milliliter, was approximately equal in aerated $\mathrm{HuSg}$ and $\mathrm{LHg}$ broth except for strain 5889 , which produced $10^{7}$ fewer CCU per milliliter in $\mathrm{LHg}$ broth in one experiment. Aeration of $\mathrm{LHg}$ broth increased the titer of all strains except 5889 by a factor between $10^{3}$ and $10^{9}$. Aeration of HuSg broth increased the titers of all strains except 4822 by a factor between $10^{0.5}$ and $10^{6.5}$

The highly passaged strain 4822 grew equally well in HuSg broth prepared with either Difco PPLO broth or Oxoid mycoplasma broth. However, with two strains at low passage, 4930 and 5889 , the number of CCU per milliliter was lower by a factor of $10^{3}$ or $10^{3.5}$ in HuSg broth prepared with the Oxoid base. On HuS agar prepared with either base, none of the seven SGM strains tested showed any difference in the number of colony-forming units (CFU) per milliliter that developed from a standard HuSg broth culture.

SGM strains grew poorly in HuS broth in which human serum was replaced by 10 to $15 \%$ rabbit serum or by $1 \%$ PPLO serum fraction (Difco). In rabbit serum broth, growth deteriorated on passage so that it was impossible to maintain cultures beyond three or four transfers. Similarly, an inoculum containng $10^{9} \mathrm{CCU} / \mathrm{ml}$, when titrated out in serum fraction broth, produced a color change only in the lowest dilutions, $10^{-1}$ and $10^{-2}$. A further titration from the $10^{-1}$ 
dilution grew only at a dilution of $10^{-1}$. The failure of strain 4822 to grow in rabbit infusion broth has already been noted in connection with antiserum production.

Morphology. In wet mounts examined by phase-contrast or dark-field microscopy or in Giemsa-stained, fixed films, SGM mycoplasmas grown in HuSg broth appeared highly filamentous. Translational motility was not detected. Electron microscopy of strains 4822 and 4788 grown in $\mathrm{HuSg}$ broth and negatively stained with ammonium molybdate confirmed the filamentous morphology of these organisms and revealed that the filaments were twisted at intervals along their length (Fig. 1), although there were no undulations of regular wavelength and amplitude as seen in spirochetes. The twisting was more obvious in preparations stained with the higher molybdate concentration $(6 \%)$. Thin sections prepared from broth cultures of 4822 and 4788 also showed that the organisms were twisted (Fig. 2 and 3). At higher magnifications, the limiting membrane was seen as a well-defined, triple-layered structure. A fairly thick, electron-dense, structureless layer was also visible outside the membrane and closely applied to it (Fig. 2, inset). The most striking feature in both strains was the appearance of striations running diagonally across many of the cell sections (Fig. 2 and 3). The striations were apparently located on the outer surface of the organisms and frequently appeared to extend beyond the cell boundary (Fig. 3). Moreover, in some sections a shadowy pattern of striations was seen in the absence of any other cell structure, suggesting that a cell or part of a cell had been cut superficially, revealing only the striations. It is possible that the striations were due to some fibrillar substance wound helically around the cells in a loose matrix of low electron density. Some evidence for the existence of such a low- density layer was seen in some sections (Fig. 3). Preparations made from colonies of 4822 and stained with sodium phosphotungstate also showed some indications of the striations (Fig. 4).

Filtration studies. A culture of strain 4822 contained $7.3 \log _{10} \mathrm{CFU} / \mathrm{ml}$ before filtration. After serial filtration through membrane filters of $650-, 450-, 300-, 220-$, and $100-\mathrm{nm}$ pore diameters, the counts were, respectively, $6.8,4.6,4.4$, 1.8 , and $<1.0 \log _{10} \mathrm{CFU} / \mathrm{ml}$. In other experiments in which the $300-n m$ filtration was omitted, passage through 650- and 450-nm filters reduced the counts by factors of approximately 10 and 100 , respectively, but no growth was detected after passage through 220 - and $100-\mathrm{nm}$ filters.

Biochemical reactions. SGM strains 4822 and 4788 produced decreases in $\mathrm{pH}$ of 1.0 to 1.3 units in glucose broth in 3 to 4 days. In the corresponding control media without added glucose, decreases of only 0.3 to $0.4 \mathrm{pH}$ units were observed, and these may have been due to traces of glucose in the serum. No changes occurred in uninoculated broth. The control cultures of $M$. pulmonis strains Kon and N14 produced pH decreases of 1.3 to 1.8 units in $40 \mathrm{~h}$. A slight decrease in $\mathrm{pH}$ of 0.2 to 0.3 units occurred in arginine broth and the corresponding control broth at $\mathrm{pH} 7.0$ when these media were inoculated with strain 4822 or strain 4788 . This was probably due to the presence of traces of glucose and was in sharp contrast to the increase of more than $1.0 \mathrm{pH}$ unit produced by $M$. subdolum within $40 \mathrm{~h}$ in arginine broth. Strain 4822 still failed to hydrolyze arginine after 10 consecutive passages at 3-day intervals or after five consecutive passages at weekly intervals in aerated HuSa or LHa broth. Strains 4822 and 4788 produced no increase in $\mathrm{pH}$ in either type of ureacontaining medium, whereas both Ureaplasma

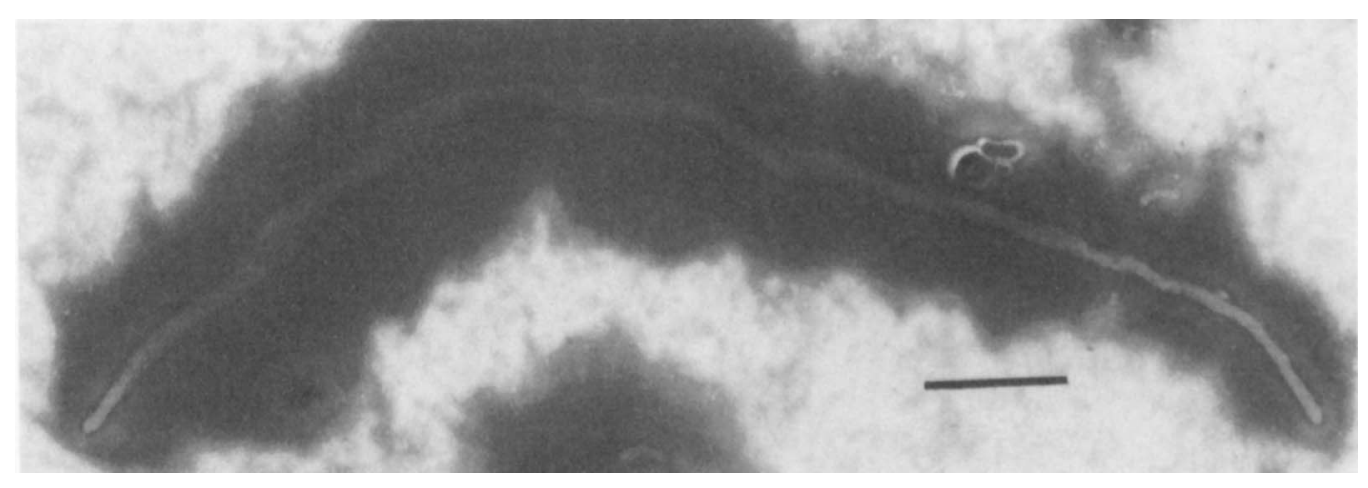

Fig. 1. Electron micrograph of strain 4788. Three-day-old culture in $20 \%$ human serum broth, fixed and negatively stained with $6 \%$ ammonium molybdate. Twisting of filament visible. Bar represents $1,000 \mathrm{~nm}$. 


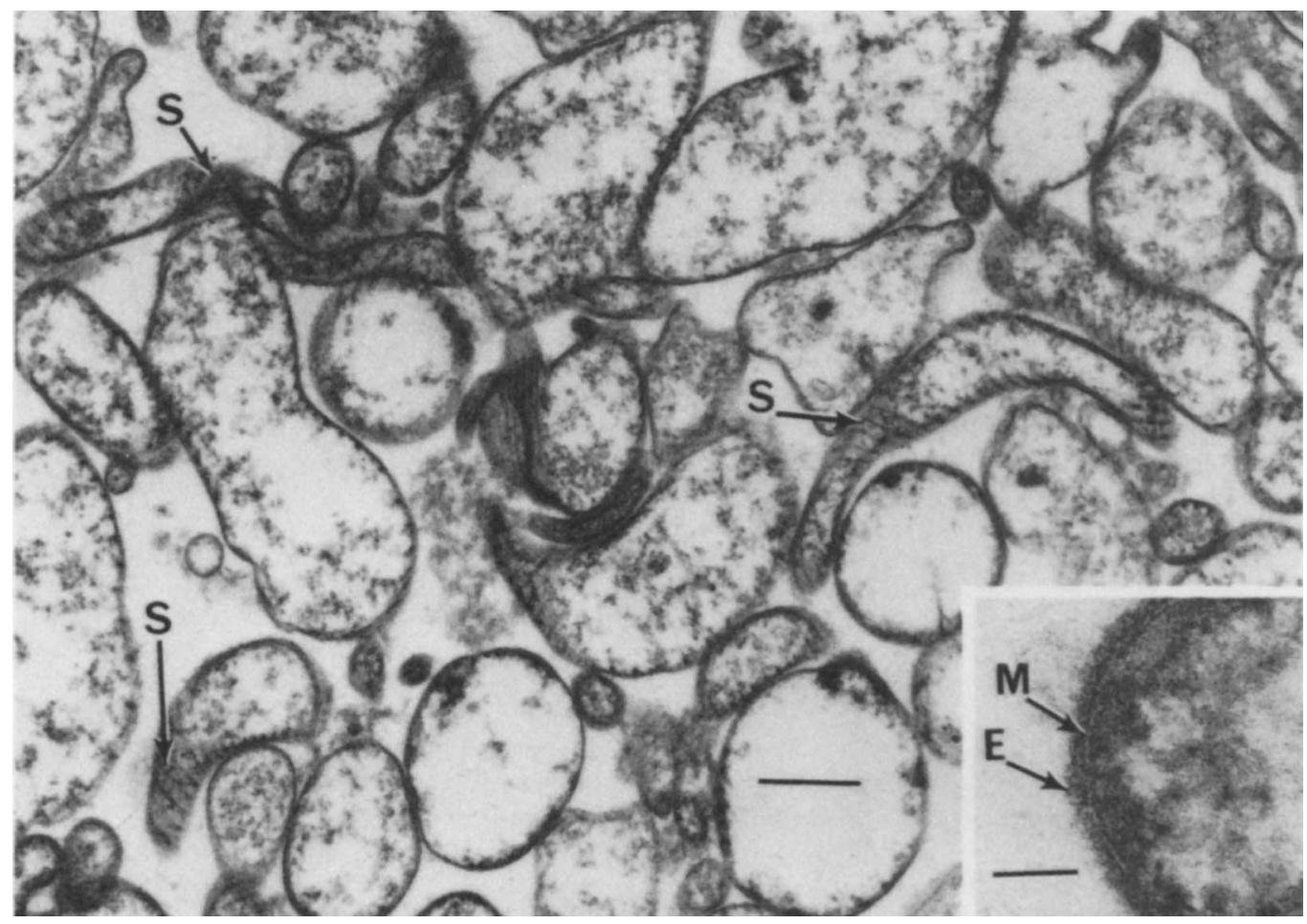

FIG. 2. Electron micrograph of strain 4822. Three-day-old culture in 20\% human serum broth, thin-section preparation stained with ruthenium red. Polymorphism and diagonal striations (S) of organisms are visible. Bar represents $400 \mathrm{~nm}$. Inset: higher magnification showing triple-layered membrane (M) and external electron-dense layer (E). Bar represents $50 \mathrm{~nm}$.

cultures gave an increase of at least $1.7 \mathrm{pH}$ units within $24 \mathrm{~h}$. The results of hemolysis, hemadsorption, and other biochemical tests are given in Table 2.

Sterol requirement. Washed filtered suspensions of strains 4822 and 4788 in PPLO broth (Difco) containing $1 \%$ bovine serum albumin fraction $V$ failed to grow on the basal sterol-free agar alone or supplemented with bovine serum albumin fraction $\mathrm{V}(0.5 \%$, wt/vol $)$, palmitic acid $(10 \mu \mathrm{g} / \mathrm{ml})$, and various concentrations of cholesterol from 0.2 to $100 \mu \mathrm{g} / \mathrm{ml}$. The same inoculum produced heavy growth on basal medium supplemented with bovine serum albumin fraction V, palmitic acid, and Difco PPLO serum fraction $(1 \%, \mathrm{vol} / \mathrm{vol})$. Growth of the eight SGM strains on HuSA was inhibited by sodium polyanethol sulfonate and digitonin. Inhibition zones with 5 and $10 \%$ sodium polyanethol sulfonate and $1.5 \%$ digitonin measured, respectively, 2 to 3,6 to 8 , and 4 to $10 \mathrm{~mm}$.

Susceptibility to antimicrobial drugs. Neither thallium acetate at $1 / 2,000,1 / 1,000$, or $1 / 500$ nor amphotericin at $2.5 \mu \mathrm{g} / \mathrm{ml}$ had any inhibitory effect on the growth of strain 4822 . This strain was, however, susceptible to the following antibiotics incorporated in dried disks: erythromycin $(15 \mu \mathrm{g})$, spiramycin $(10 \mu \mathrm{g})$, oleandomycin $(15 \mu \mathrm{g})$, streptomycin $(25 \mu \mathrm{g})$, neomycin $(10 \mu \mathrm{g})$, kanamycin $(5 \mu \mathrm{g})$, novobiocin $(5 \mu \mathrm{g})$, spectinomycin $(25 \mu \mathrm{g})$, and tetracycline $(10 \mu \mathrm{g})$. Strain 4822 was completely insensitive to disks containing colistin $(50 \mu \mathrm{g})$ and polymyxin B (300 $\mu \mathrm{g})$, but there was a zone of partial inhibition around disks containing sulfamethoxazole-trimethoprim $(25 \mu \mathrm{g})$. Tests in HuSg broth indicated that the eight SGM strains were susceptible to erythromycin at concentrations as low as $0.1 \mu \mathrm{g} / \mathrm{ml}$. This concentration of erythromycin reduced the titer by about $10^{7} \mathrm{CCU} / \mathrm{ml}$ compared with a control culture titrated in the absence of the antibiotic (13).

Serological results. In GI tests on HuS or LH agar, the eight SGM strains were inhibited by the two antisera to strain 4822 , giving zones 5 to $10 \mathrm{~mm}$ wide depending on the medium, the thickness of the agar, and the passage level at which the strains were tested. Zones of inhibition 


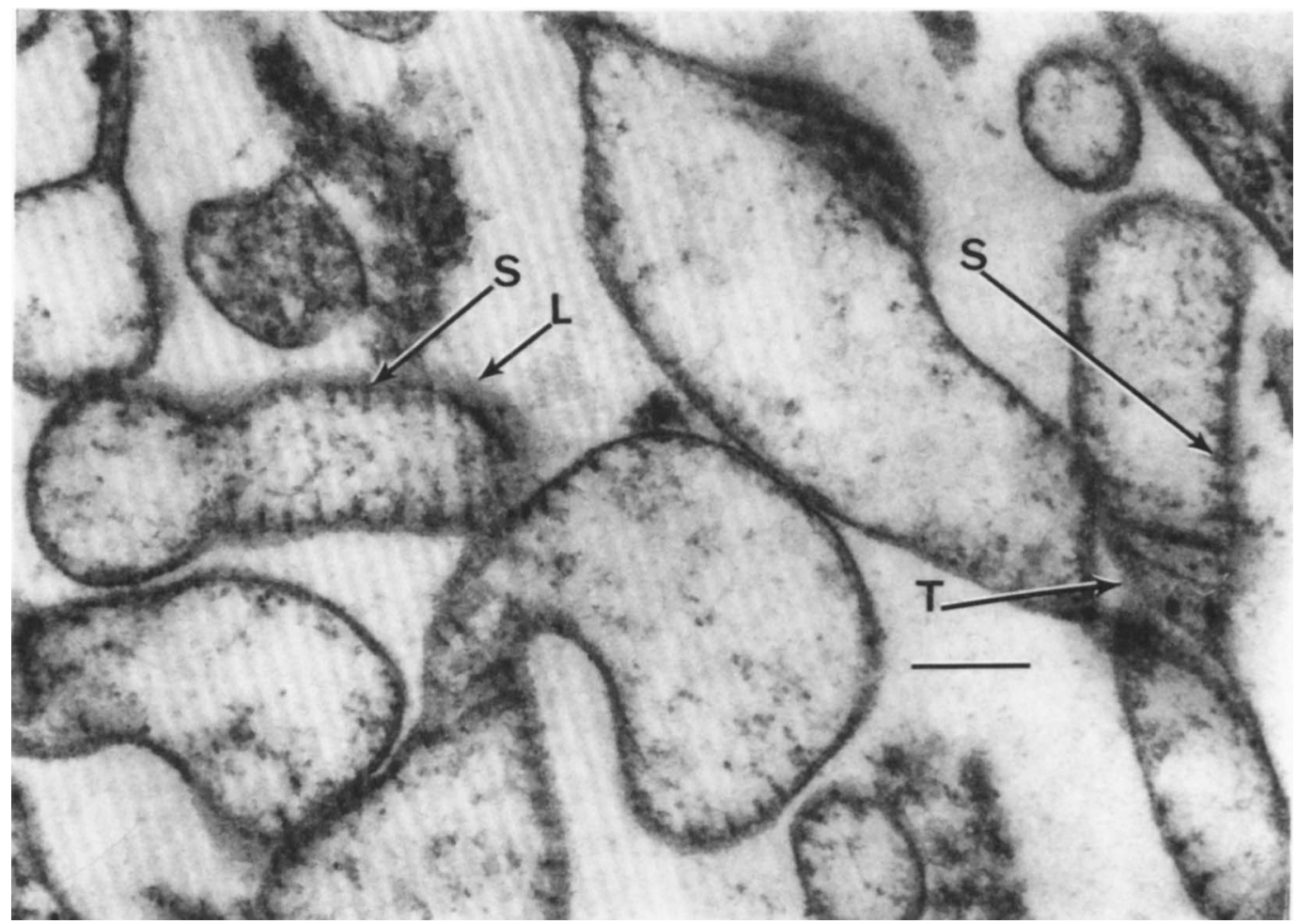

FIG. 3. Electron micrograph of strain 4788. Four-day-old culture in $20 \%$ human serum broth, thin-section preparation stained with ruthenium red. Striations (S) are visible overlying the cell boundary and, apparently, an external layer of low electron density $(L)$. A filament twisted at its center $(T)$ is also shown. Bar represents $200 \mathrm{~nm}$.

were narrower when strains had undergone a small number $(<20)$ of in vitro transfers. In addition, two lines of precipitate developed at the edge of the inhibition zone, except when growth was very heavy and confluent, and persisted even after prolonged incubation.

In accordance with the principles laid down by the Subcommittee on the Taxonomy of the Mycoplasmatales (18), the most extensive serological comparisons were made with those species of Mycoplasma found in the same host or those having the same metabolic characteristics, in this case the ability to ferment glucose.

In GI tests on HuS or LH agar, in which strain 4822 was compared in both directions with the 59 species listed in Table 1, no cross-reactions were observed except with certain strains of $M$. pulmonis on LH agar. On this medium, strain 4822 was inhibited by antisera against $M$. pulmonis strains $\mathrm{M} 1$ and Kon, and $M$. pulmonis strains M1, N14, and Ash were inhibited by 4822 antiserum $\mathrm{A}$ but not by antiserum $\mathrm{B}$. No crossreactions were observed on $\mathrm{HuS}$ agar. This cross-reaction was subsequently investigated in greater detail (see next section).
In IFA tests performed in both directions, no cross-reactions were observed between strain 4822 and 33 glycolytic species, 4 species of nonglycolytic, non-arginine-hydrolyzing mycoplasmas, the 2 arginine-hydrolyzing mycoplasmas found in horses ( $M$. equirhinis and $M$. subdolum), and Spiroplasma citri.

Five other SGM strains (4788, 4791, 4927, 4929 , and 5173) were also tested on HuS agar by the GI method against antisera to 20 species of glycolytic mycoplasmas including $M$. pulmonis. No inhibition was observed.

In addition to GI and IFA, the MI technique was used for testing five taxa (M. mycoides subsp. mycoides, $M$. mycoides subsp. capri, $M$. agalactiae, $M$. suipneumoniae, and $M$. floccu. lare) against 4822 antiserum $B$. No inhibition was observed at the lowest serum dilution tested.

Serological comparison of strain 4822 and $M$. pulmonis. The cross-reaction of strain 4822 with $M$. pulmonis was investigated by GI and MI tests with both $\mathrm{HuS}$ and $\mathrm{LH}$ media and also by the IFA test on colonies grown on HuS agar. GI tests of strain 4822 against five $M$. pulmonis antisera and of four strains of $M$. pul. 


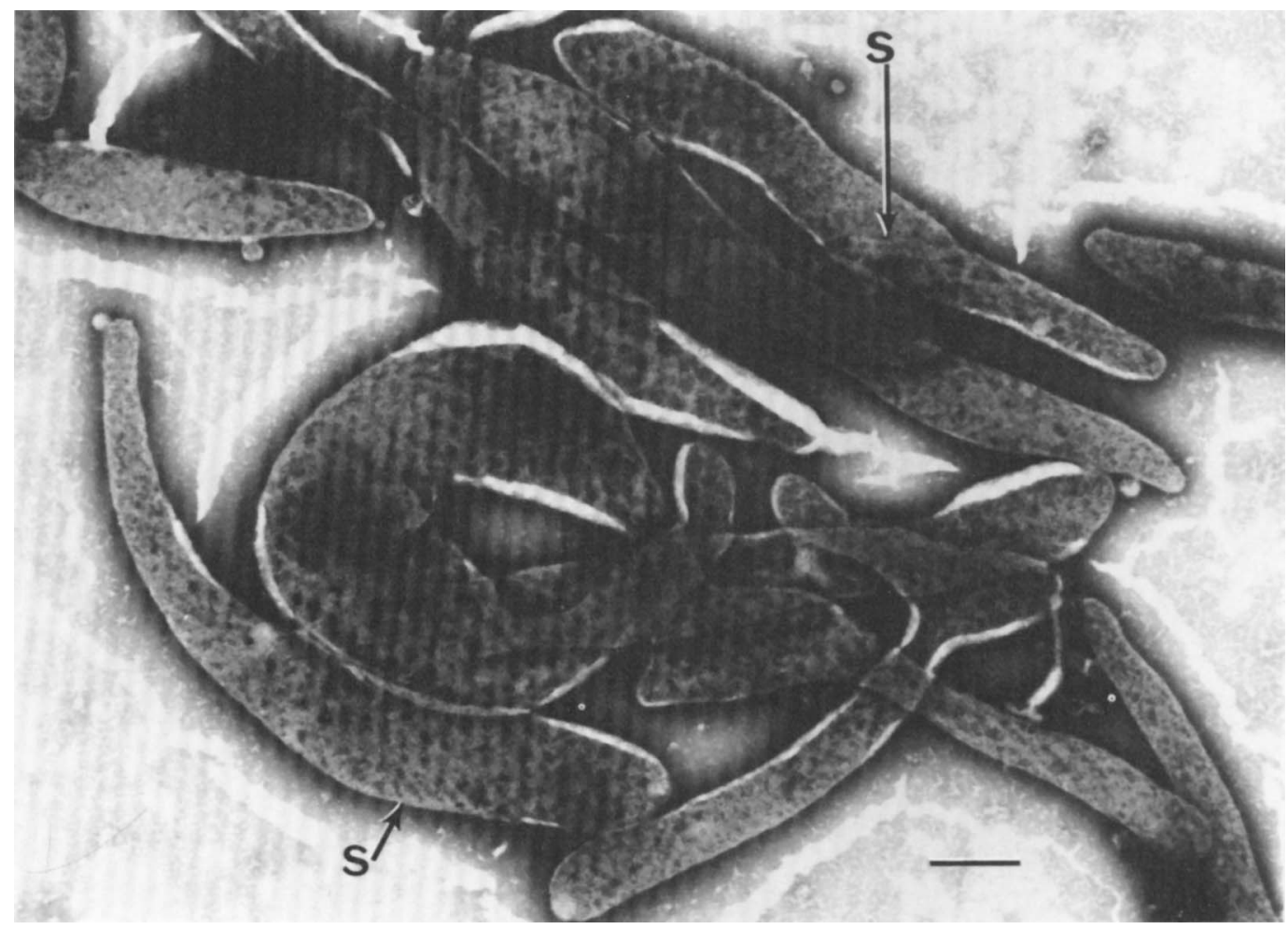

FIG. 4. Electron micrograph of strain 4822 grown on $20 \%$ human serum agar, fixed, and negatively stained with sodium phosphotungstate. Diagonal striations (S) are visible on some cells. Bar represents $400 \mathrm{~nm}$.

TABLE 2. Biochemical reactions of SGM strains 4822 and 4788

\begin{tabular}{|c|c|}
\hline Characteristic & Reaction \\
\hline Glucose metabolism & $+(\text { Acid })^{a}$ \\
\hline Arginine hydrolysis & - \\
\hline Urea hydrolysis ....... & - \\
\hline Tetrazolium reduction $^{b}$ & - \\
\hline Tellurite reduction ${ }^{c} \ldots$ & - \\
\hline Phosphatase activity & - \\
\hline Catalase activity ..... & - \\
\hline Oxidase activity & - \\
\hline Film formation. & + \\
\hline \multicolumn{2}{|l|}{ Hemolysis of erythrocytes } \\
\hline Sheep .................. & $\alpha$ or $\alpha^{\prime}$ \\
\hline Horse. & $\alpha^{\prime}$ or $\beta$ \\
\hline \multicolumn{2}{|l|}{ Hemadsorption } \\
\hline Sheep and guinea pig erythrocytes & + \\
\hline
\end{tabular}

${ }^{a}$ Slow.

${ }^{b}$ Tested in fluid and on solid media.

${ }^{c}$ Potassium tellurite $(0.0002 \%)$ in fluid and solid media; $0.005 \%$ Potassium tellurite in solid medium. Some inhibition of growth on solid medium at both concentrations.

monis against 4822 antisera $A$ and $B$ revealed no inhibition when performed on HuS agar (Table 3 ). In similar tests on LH agar, 4822 was significantly inhibited by two out of five antisera to $M$. pulmonis (M1 and Kon), and three out of four strains of $M$. pulmonis (N14, Ash, and M1) were inhibited by 4822 antiserum $A$ but not by antiserum B (Table 3 ). The sizes of the inhibition zones on LH media were quite variable. In some tests the zones comprised only 0.5 to $2.0 \mathrm{~mm}$ of partial inhibition, and such zones are not considered to be significant. No preimmunization serum corresponding to 4822 antiserum $\mathrm{A}$ was available, but that corresponding to 4822 antiserum B also inhibited $M$. pulmonis on $\mathrm{LH}$ but not on HuS agar (Table 3). Preimmunization sera corresponding to the antisera against $M$. pulmonis strains N14, Kon, and MB were also available, but none inhibited strain 4822 or any of the $M$. pulmonis strains on LH or HuS agar.

MI tests performed in $\mathrm{HuSg}$ broth revealed no cross-reactions between strain 4822 and several strains of $M$. pulmonis (Table 4). In similar tests in $\mathrm{LHg}$ broth, 4822 was inhibited by antiserum to $\mathrm{N} 14$ at titers of $1 / 320$ to $1 / 640$. The other $M$. pulmonis antisera inhibited 4822 at titers of $1 / 40$ or less. In all cases, the titers of strain 4822 were lower than those of the homologous strain (Table 4). Although the four $M$. pulmonis strains were inhibited by 4822 antiserum $\mathrm{A}$ in GI tests on LH agar, the corresponding titers in the MI test on LHg broth were never $>1 / 20$. 
TABLE 3. Comparison of SGM strain 4822 and M. pulmonis strains in growth inhibition tests on two media

\begin{tabular}{|c|c|c|c|c|c|c|c|c|c|}
\hline \multirow{3}{*}{ Strain } & \multirow{3}{*}{ Medium } & \multicolumn{8}{|c|}{ Inhibition zone $(\mathrm{mm})^{a}$ with serum against: } \\
\hline & & \multicolumn{3}{|c|}{ Strain 4822} & \multicolumn{5}{|c|}{ M. pulmonis strains: } \\
\hline & & Antiserum A & Serum $B(N)^{b}$ & Antiserum B & N14 & Ash & M1 & Kon & MB \\
\hline \multirow[t]{2}{*}{ SGM strain 4822} & LH & 8-10 (pr) ${ }^{c}$ & 0 & $8-10(\mathrm{pr})^{c}$ & 0 & 0 & 9 & $3-4$ & 0 \\
\hline & HuS & $7-10$ & 0 & $8(p r)^{c}$ & 0 & 0 & 0 & 0 & 0 \\
\hline \multirow{2}{*}{ M. pulmonis N14 } & LH & $0-5$ & $4-5$ & 0 & 4-6 & $5-7$ & $6-8$ & $4-6$ & $7-8$ \\
\hline & HuS & 0 & 0 & 0 & 4-6 & $4-7$ & 5 & 4 & $6-9$ \\
\hline \multirow[t]{2}{*}{ M. pulmonis Ash } & $\mathrm{LH}$ & $0-3(\mathrm{pi})^{d}$ & $3-5(\mathrm{pi})^{d}$ & 0 & 2 & $6-7$ & 5 & $6-7$ & $5-7$ \\
\hline & HuS & 0 & 0 & 0 & $6-8$ & $7-8$ & 8 & 8 & $6-9$ \\
\hline \multirow[t]{2}{*}{ M. pulmonis $\mathrm{M} 1$} & $\mathrm{LH}$ & $2-4$ & $0-3(\mathrm{pi})^{d}$ & 0 & $3-4$ & 3 & $7-8$ & 5 & $\mathrm{NT}^{e}$ \\
\hline & HuS & 0 & 0 & 0 & $3-5$ & 3 & 8-9 & 5 & $6-10$ \\
\hline \multirow[t]{2}{*}{ M. pulmonis Kon } & LH & 0 & $0-5(\mathrm{pi})^{d}$ & 0 & 0 & 6 & 5 & 10 & 6 \\
\hline & Hus & 0 & 0 & 0 & 0 & $6-7$ & $5-7$ & 9 & $7-10$ \\
\hline
\end{tabular}

\footnotetext{
${ }^{a}$ Measured from edge of disk. Range of measurements in two to six replicate tests. Results with preimmunization sera corresponding to N14, Kon and MB were all negative; others were not available. Data in boldface are homologous reactions.

${ }^{b}$ Pre-immunization serum corresponding to 4822 antiserum $B$.

${ }^{c} \mathrm{pr}$, Presence of two precipitin lines at edge of inhibition zone.

${ }^{d}$ pi, Partial inhibition; reduction in number of colonies within zone.

NT, Not tested.
}

In IFA tests, strain 4822 and $M$. pulmonis strains N14, M1, Ash, and Kon were titrated against 4822 antisera $A$ and $B$; the preimmunization serum from rabbit B; antisera to $M$. pulmonis N14, M1, Kon, and MB; and the preimmunization sera from rabbits immunized with N14, Kon, and MB. All the preimmunization sera had titers of $<1 / 20$, the lowest dilution tested. Homologous titers ranged from $1 / 320$ to $1 / 2,560$. Titers of strain 4822 with all the $M$. pulmonis antisera were $<1 / 20$. Similarly, titers of the four M. pulmonis strains were $<1 / 20$ with both antisera against 4822 . Thus, no cross-reactions between strain 4822 and several strains of M. pulmonis were demonstrable by the IFA test.

\section{DISCUSSION}

The equine SGM strains were typical Mollicutes in possessing a triple-layered limiting membrane, in colonial morphology, in filterability through a membrane filter with a pore diameter of $450 \mathrm{~nm}$, and in failing to revert to a bacterial form (18). As with most mycoplasmas, they failed to produce catalase or oxidase. A requirement by them for cholesterol could not be shown by the standard method, the two strains tested failing to grow in media in which serum was replaced by bovine serum albumin fraction V, palmitic acid, and cholesterol. The substitution of PPLO serum fraction for cholesterol in this medium resulted in heavy growth. Serum fraction contains cholesterol, but in this case it clearly supplied some other, unidentified component(s) required by these equine mycoplasmas. Nevertheless, the stimulation of growth by serum fraction and the inhibition produced by sodium polyanethol sulfonate and digitonin suggest that the SGM strains are sterol dependent and that they are therefore members of Mycoplasmataceae or Spiroplasmataceae.

The tendency of the SGM strains to form filaments, the apparent twisting of the filaments, and the diagonal striations were unusual morphological features. Despite the twisting, however, phase-contrast microscopy of live cells revealed no helical morphology like that of Spiroplasma spp. The striations may be superficial, helically wound fibrils around the cell external to the membrane. Freeze-etching might determine their position more precisely. It would also be of interest to examine the SGM strains by microcinematography. Fibrillar, actin-like protein has been reported in $M$. pneumoniae (14), a mycoplasma known to execute contractile and gliding movements.

Another characteristic of the SGM mycoplasmas was their nutritional fastidiousness. On primary isolation and during early passages at least, their growth on HuS agar was superior in several respects to that on LH agar. These growth characteristics of the SGM mycoplasmas in HuS and LH media confirm our original suggestion (15) that their isolation from nasopharyngeal swabs was facilitated by the use of HuS media which were not employed in earlier surveys of the mycoplasmal flora in the equine respiratory tract. Both $\mathrm{LH}$ agar and $\mathrm{LHg}$ broth sustained growth poorly, and the metabolism of glucose appeared to be strongly suppressed unless the broth was aerated during incubation. This fastidious character in early passages after isolation. was also shown by the preference of the strains, 


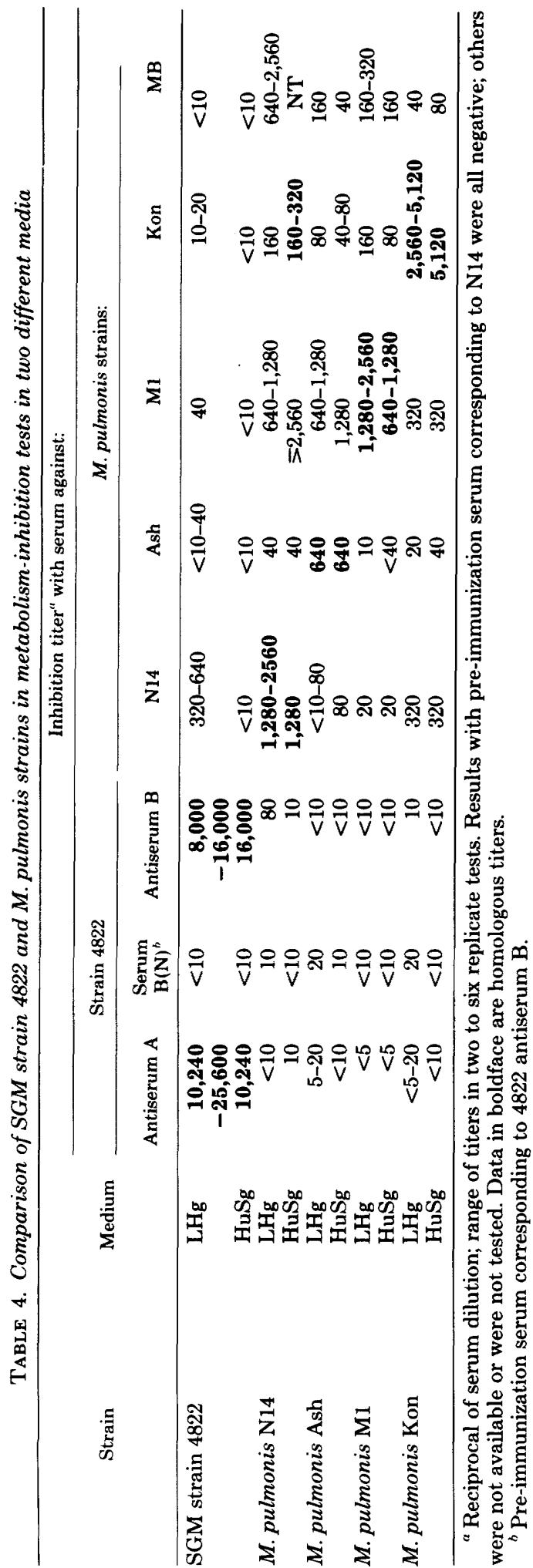

especially 5889 , for one of two broth bases and their failure to adapt easily to other types of serum supplement or to grow in a strictly anaerobic atmosphere. Since the SGM mycoplasmas produced only a slow and somewhat restricted reduction in the $\mathrm{pH}$ of glucose-containing media, more sophisticated enzyme studies are required to establish that these organisms do indeed ferment glucose. In contrast to the glycolytic species examined by Aluotto et al. (2), the SGM strains did not reduce tetrazolium or tellurite either aerobically or anaerobically. However, because the SGM strains were inhibited by $0.02 \%$ tetrazolium (Aluotto et al. [2]), they had to be tested at $0.005 \%$. Similarly, tellurite at just under half the recommended concentration (2) was slightly inhibitory. Isolated colonies of the SGM strains strongly adsorbed erythrocytes and lysed equine erythrocytes. In these respects the SGM strains resemble $M$. pneumoniae. Most species of Mycoplasma are resistant to erythromycin at concentrations of 10 to $100 \mu \mathrm{g} / \mathrm{ml}(13)$, but the SGM strains were very susceptible to this drug, a feature they share with $M$. pneumoniae (17) and $M$. gallisepticum, which are pathogens of the human and avian respiratory tracts, respectively.

That the SGM strains constitute a group which is distinct from 58 other recognized species of Mycoplasma and S. citri is confirmed by the serological evidence. The inhibition of strain 4822 by some $M$. pulmonis antisera in GI and MI tests and of $M$. pulmonis by 4822 antiserum $A$ and pre-immunization serum B in GI tests on LH media is thought to be nonspecific for the following reasons. First, not all the antisera inhibited the heterologous species. Second, one pre-immunization serum was also inhibitory. Last, the inhibitory effect was observed only on LH medium, which was suboptimal for the growth of SGM strains and also for M. pulmonis strain N14 (J. Poland, unpublished data). The effect was never observed with HuS media. Nonspecific inhibitory factors for mycoplasmas occur in some rabbit sera. Therefore the inhibition probably arose from two factors: (i) the effect of nonspecific inhibitors in some sera, and (ii) the inhibitory nature of LH media; some batches of LH media appeared more toxic than others, the sizes of the heterologous inhibition zones varying considerably in different experiments. No crossreactions between strains 4822 and $M$. pulmonis were detectable by either GI or MI when HuS media were used, or by IFA, a test which does not depend on specific inhibition. These results emphasize the problems of using serological tests based on inhibition of growth by specific antibody for the identification or classification of 
fastidious or slow-growing mycoplasmas and underlines the advisability of using additional tests such as IFA or complement fixation.

The importance of SGM strains in the equine respiratory tract is obscure. Although the isolation rate was low, high levels of MI antibody were found in other horses in the three stables from which the isolates were recovered (15). Hence, the organisms may have been more widespread than indicated by the isolation rate. Although SGM strains certainly exhibit some of the properties shown by mycoplasmas pathogenic for the respiratory tracts of other hosts, their pathogenicity in horses is unknown and their isolation was not related to episodes of clinical respiratory disease (15). Moreover, strain 4822 failed to cause clinical disease in two formerly gnotobiotic foals that had been kept in isolation, although it persisted for several weeks in the nasal cavity and evoked a strong antibody response (15). Perhaps intranasal instillation is unsatisfactory for producing respiratory disease, as is the case with $M$. mycoides subsp. mycoides.

The morphological, biological, and serological properties of the SGM strains suggest that they comprise a new species. Although their unique morphology distinguishes them from other $M y$. coplasma species, they do not possess the regular helical symmetry of spiroplasmas nor are they serologically related to $S$. citri, the only species of Spiroplasma currently recognized. Moreover, unlike spiroplasmas, SGM mycoplasmas were isolated from a vertebrate instead of a plant, insect, or arthropod host and were able to colonize the equine respiratory tract. Even the suckling mouse cataract agent (spiroplasma), which is pathogenic for vertebrates, was originally isolated from a pooled extract of rabbit ticks. It is therefore suggested that the SGM strains be classified in the family Mycoplasmataceae. The name Mycoplasma fastidiosum sp. nov. is proposed (fas.tid.i.o'sus. L. adj. fastidiosus, fastidious, referring to the nutritionally fastidious nature of the organism on primary isolation). Strain 4822 is the type strain of $M$. fastidiosum. A culture of this strain has been deposited in the National Collection of Type Cultures (NCTC), London, where it has been given the number 10180 .

\section{ACKNOWLEDGMENTS}

Part of this work was carried out at the Lister Institute of Preventive Medicine, London SW1 8RH, England and was supported by a grant from the Horserace Betting Levy Board, London, W.C. 1, England.

We are grateful to those workers who so generously provided antisera and cultures, and in particular to $R$. H. Leach (Mycoplasma Reference Laboratory, Norwich, England) who also carried out serological tests with a number of strains not available to us. We also thank P. Whittlestone (School of Veterinary Medicine, Cambridge, England) for carrying out GI and MI tests on certain species pathogenic for animals, I. M. Smith (Royal Veterinary College) for advice on the manuscript, and A. P. Bland (A.R.C. Institute for Research on Animal Diseases, Compton, England) for performing the electron microscopy. Jill Lewis (Royal Veterinary College) and Carol Robinson (Lister Institute) gave valuable technical assistance.

\section{REPRINT REQUESTS}

Address reprint requests to: Dr. R. M. Lemcke, ARC Institute for Research on Animal Diseases, Compton, Newbury, Berkshire, England.

\section{LITERATURE CITED}

1. Allam, N. M., and R. M. Lemcke. 1975. Mycoplasmas isolated from the respiratory tract of horses. J. Hyg. 74: 385-408.

2. Aluotto, B. B., R. G. Wittler, C. O. Williams, and J. E. Faber. 1970. Standardized bacteriologic techniques for the characterization of Mycoplasma species. Int. J. Syst. Bacteriol. 20:35-58.

3. Clyde, W. A. Jr. 1964. Mycoplasma species identification based upon growth inhibition by specific antisera. J. Immunol. 92:958-965.

4. Edward, D. G. ff. 1971. Determination of sterol requirement for Mycoplasmatales. J. Gen. Microbiol. 69:205210.

5. Freundt, E. A., B. E. Andrews, H. Ernø, M. Kunze, and F. T. Black. 1973. The sensitivity of Mycoplasmatales to sodium polyanethol sulphonate and digitonin. Zentralbl. Bakteriol. Parasit. Infektionskr. Hyg. Abt. 1 Orig. Reihe A 225:104-112.

6. Gourlay, R. N., S. G. Wyld, and R. H. Leach. 1977. Mycoplasma alvi, a new species from bovine intestinal and urogenital tracts. Int. J. Syst. Bacteriol. 27:86-96.

7. Howard, C. J., and R. N. Gourlay. 1974. An electronmicroscopic examination of certain bovine mycoplasmas stained with ruthenium red and the demonstration of a capsule on Mycoplasma dispar. J. Gen. Microbiol. 83:393-398.

8. Howard C. J., R. N. Gourlay, and J. Collins. 1975. Serological comparison between twenty-five bovine Ureaplasma (T-mycoplasma) strains by immunofluorescence. Int. J. Syst. Bacteriol. 25:155-159.

9. Kirchhoff, H. 1974. Neue spezies der Fam. Acholeplas mataceae und der Fam. Mykoplasmataceae bei Pferden. Zentralbl. Veterinaermed. Reihe B 21:207-210.

10. Kirchhoff, H. 1978. Mycoplasma equigenitalium, a new species from cervix region of mares. Int. J. Syst. Bacteriol. 28:496-502.

11. Lemcke, R. M., and N. M. Allam. 1974. Some problems arising during the characterization of mycoplasmas from the equine respiratory tract. Coll. Inst. Natl. Santé Rech. Med. 33:153-160.

12. Lemcke, R. M., and H. Kirchhoff. 1979. Mycoplasma subdolum, a new species isolated from horses. Int. J. Syst. Bacteriol. 29:42-50.

13. Lewis, J., and J. Poland. 1978. Sensitivity of mycoplasmas of the respiratory tract of pigs and horses to erythromycin and its use in selective media. Res. Vet. Sci. 24:121-123.

14. Niemark, H. C. 1977. Extraction of an actin-like protein from the prokaryote Mycoplasma pneumoniae. Proc. Natl. Acad. Sci. U.S.A. 74:4041-4045.

15. Poland, J., and R. M. Lemcke. 1978. Mycoplasmas of the respiratory tract of horses and their significance in upper respiratory tract disease. Proc. 4th Int. Conf. Equine Infect. Dis. Lyon, 1976. J. Equine Med. Surg. 
Suppl. 1:438-446.

16. Rosendal, S., and F. T. Black. 1972. Direct and indirect immunofluorescence of unfixed and fixed mycoplasma colonies. Acta Pathol. Microbiol. Scand. Sect. B 80: 615-622.

17. Slotkin, R. L., W. A. Clyde, Jr., and F. W. Denny. 1976. The effects of antibiotics on Mycoplasma pneumoniae in vitro and in vivo. Am. J. Epidemiol. 86:225237.

18. Subcommittee on the Taxonomy of Mollicutes. 1979.
Proposal for minimal standards for descriptions of new species of the class Mollicutes. Int. J. Syst. Bacteriol. 29:172-180.

19. Taylor-Robinson, D., R. H. Purcell, D. C. Wong, and R. M. Chanock. 1966. A colour test for the measurement of antibody to certain mycoplasma species based upon the inhibition of acid production. J. Hyg. 64:91104.

20. Windsor, G. D. 1973. The isolation of Mycoplasma from horses. Vet. Rec. 93:593-594. 